\title{
Desviación del crecimiento y desarrollo del embrión o feto según estado nutricional materno
}

DR. LUIS GIVOVICH M. *.

En una conferencia que en 1970 dictara $\mathbf{C} . \mathbf{M}$. Drillien,, sostuvo que la escasez aguda y grave de alimentos parece motivar en la mujer incapacidad para concebir, y aumentos de abortos tempranos, y no disminución del peso de nacimiento de los productos que sobreviven lo suficiente para ser viables. No fue una nueva aseveración. Ya lo habían expresado en igual forma muchos otros investigadores; pero lo trascendente es que una variación de los pesos promedios de nacimiento fue considerada sólo como derivada de influencia nutricional, en un simposium sobre "el neonato pequeño para la edad de gestación", y así quedó expresado en el texto de las clínicás pediátricas que lo recopiló. Numcrosas publicaciones posteriores en el país y en el extranjero han continuado con las mismas observaciones en las que C. M. Drillen apoyó su tesis e interpretación. La observación de Gruenwald (1) que comparó los pesos promedios de nacimiento de los niños japoneses durante los años de pobreza difundida de la post-guerra, con los de la riqueza relativa del sexto decenio; y aquella otra de Smith (2) que comparó los pobres incrementos promedios de peso de las embarazadas durante la hambruna de Rotterdam, habida en el invierno de 1944, con la relativa muy baja disminución de los pesos promedios de los hijos concebidos; y muchas otras observaciones de hambrunas o experiencias (3-45-6-7) de disponibilidades dietéticas deficitarias en poblaciones que no muestran una disminución significativa paralela de los incrementos ponderales de las embarazadas, con respecto a los pesos promedios de nacimiento de los recién nacidos, han permitido extraer esta controversial conclusión a expositores que no han sopesado una realidad irredargüible: A medida que el nivel socio-económico

\footnotetext{
- Minisclerio te Sajud. Of. cle Salud Infantil.
}

de las poblaciones se deteriora la desnutrición hace presa de ellas y entre otros los índices de desnutrición fetal intrauterina se elevan considerablemente. En esas observaciones y experiencias estos índices no fueron evaluados porque al parecer consideeraron se expresaban con una disminución de los pesos promedios de nacimiento, olvidándose que los promedios no manifiestan los rangos de variación extremos que se compensan aritméticamente para aminorarse en un promedio; que sólo aparece significativo cuando el porcentaje de deterioro es muy elevado. Si en las experiencias relatadas se hobiesen considerado los índices de desnutrición intrauterina, o de cualquiera otra desviación del crecimiento, seguramente se habría llegado a una muy diferente conclusión, no sólo por lo dicho sino porque además habria obligado a determinar la edad gestacional de cada recién nacido; to que es básico para poder valorar sus estados nutritivos (8-9). Si el bajo peso es más consecuencia de una interrupción del crecimiento por un parto prematuro que de una aiteración propiamente tal es una interrogante que no puede quedar albierta si al peso se le está considerando como índice de daño nutricional.

En la probabilidad de desviaciones del crecimiento y desarrollo fetal intervienen además una cadena de variables ecológicas, condicionantes de la respuesta a cualquiera noxa determinante de alguna influencia negativa, que hace aún más dificil extraet conclusiones valederas, tales como: paridad, períodos de infertilidad, talla edad y estado nutritivo prevto de las madres, manejo de las embarazadas para la corrección oportuna y eficiente de su patologia, prevenir el parto prematuro, procurar cl suministro adecuado de nutrientes o evitar la fatigabilidad física o psíquica, estado de madurez, sexo y constitución genética fetal, etc. Es 
indudable que estos factores deben ser también estimados en cualquier estudio comparativo de medidas antropométricas de neonatos para que las conclusiones no resulten discrepantes. Por ello es que las conclusiones obtenidas de las observaciones en discusión son objetables.

Ahora bien, si lo que se pretende demostrar es la influencia de la mala nutrición materna en el determinismo de desviaciones de este proceso biológico de crecimiento fetal intrauterino se hace indispensable establecer previamente cuál es la ingesta cualicuantitativa mínima requerible por la embarazada, tomando en cuenta los requerimientos extras de la gravidez, y los procesos metabólicos de adaptación que se desencadenan para favorecer un ahorro proteico, a través de un transporte activo-útero-placentario. Sólo así y aunque sea en forma convencional podremos precisar una condición carencial, y en todo caso sin olvidar que la disponibilidad o consumo total de alimentos de una población, promediada en un universo, no es representativa del consumo individual. Sienpre habrá grupos con muy altas o bajas ingestas.

Las observaciones relatadas se hacen también objetables porque sólo se determinaron promedios de ingesta calórico global y/o proteica, y no se estableció para la comparación una ingesta mínima exigible para ponderar déficits por debajo de ella.

En suma como las experiencias y observaciones expuestas se han basado sólo en promedios de pesos y de ingestas, no han considerado si las desviaciones son por prematuridad verdadera o alteración propiamente tal del crecimiento y desarrollo embrionario o fetal, ni tampoco estimaron la influencia de variables condicionantes, las conclusiones son tan controversiales que no pueden seguir sirviendo de punto de apoyo a una aseveración no suficientemente avalada.

Si como contrapartida se pretendiese demostrar a través de observaciones epidemiológicas, o experimentalmeente, que la nutrición materna ticne una real influencia sobre este proceso biológico de crecimiento y desarrollo intrututerino, y la carencia nutricional en el determinismo de las desviaciones que pudieren acontecer, debemos no sólo estimar las objeeciones metológicas, e interferencias de las variables mencionadas, sino que además y muy fundamentalmente uniformar los criterios para precisar y diagnosticar las desviaciones fisiológicas y los deterioros, tanto en la madre como en el hijo. Ello exige aceptar previamente una metódica que valore las variaciones fisiológicas y probabilidades mórbidas a que queda sometida la constitución del zigoto y su posterior crecimiento y desarrollo, hasta dar forma a un ser especificamente definido y apto para nacer a la vida extrauterina, en un período de tiempo diverso pero predeterminado según sea la especie.
Excluyendo los factores genéticos, que te dan al zigoto una característica de crecimicnto específico y heredítario, todos los otros son dependientes del ambiente en el que este proceso se realiza, y del cumplimiento o no del período de tiempo que específicamente debe disponer para cumplirlo. Si el ambiente intrauterino es desfavorable habrá un deterioro subsecuente que se manifestará en alguna alteración del crecimiento y desarrollo. Si el tiempo no es suficiente o se sobrepasa habrá una desviación fisiológica, expresada en signos somáticos o funcionales de inntadurez o post-madurez, que evidencia una interrupción o prolongación del crecimiento y desarrollo, pero no una alteración propiamente tal. Ambas condiciones conducen por diferentes caminos a una desviación de las medidas ponderales, y esa similitud semiológica induce a error si no se esclarece. Así si se pretende clasifificar a los recién nacidos de acuerdo a la madurez alcanzada en: "prematuros" y de "término", y para hacerlo sólo se recurre a la estimación del peso de nacimiento, se comete el error de considerar como prematuros a un buen porcentaje de R. Nacidos orgánica y funcionalmente ya maduros, y por tanto falsos prematuros no obstante tener un peso inferior a 2.500 grs., y como de "término" a muchos que orgánica y funcionalmente son inmaduros y en consecuencia verdaderos prematuros. Al revés, estimar como una alteración del crecimiento y desarrollo medidas ponderales interiores a 2.500 grs., es otro error si no se precisa previamente si ese menor peso es sólo derivado de una condición fisiológica de inmadurez fetal. La clasificación internacional de los R. Nacidos en: "prematuros" y de "término" adolece de estos defectos y por ello ha caído en descrédjto. Debe llegarse a un acuerdo en la nomenclatura más consecuente con lo que se quiere expresar. Lo con. trario acarrea confusión e imposibilidad de precisar la condición del sujeto que se refiere. Mientras ilegue hemos optado por clasificarlos en: "maduros" e "inmaduros", adjetivándoles de "desnutridos" si no tienen el peso correspondiente a su edad gestacional (8-9).

Esclarecer si la causa de una desviación ponderal es por interrupción o por alteración del crecimiento y desarrollo exige tanto una metódica expedita y uniforme para valorar la edad gestacional. $\mathrm{Si}$ consideramos que la estimación de un daño nutricional se obtiene exigiendo acordados patrones de mortalidad ponderal para cada edad gestacional, se hace también necesario un acuerdo previo acerca de los patrones de normalidad a aplicar en la metódica diagnóstica de un deterioro. Ni lo uno ni lo otro ha ocurrido hasta la fecha y por esta razón ni siquiera los índices de $R$. Nacidos de bajo peso para la edad gestacional, que señalan diversas publicaciones, son confiables para un estudio comparativo. Esta anarquía impe- 
ránte en las metódicas para valorar la edad gestacional, $y$ estimar los patrones de normalidad mús adecuados para diagnosticar las desviaciones, se pone en evidencia en los discrepantes porcentajes de desnutrición que señalan paíscs con muy similar situación socio-económica y aún áreas dentro de una misma ciudad (9-10).

No sólo se hace criticable toda observación o experiencia que pretenda medir la influencia de la nutrición dal R. N. en base a promedios ponderales; o a porcentajes de desviaciones obtenidos con diferentes metodologías, sino también cuando no se considera que ellas pueden evidenciarse no sólo en alteraciones del crecimiento in toto sino tambićn localizadas en diversos órganos o sistemas, y que se manifiestan en diversas malformaciones, disfun funciones o neoformaciones con que el neonato puede nacer. Estas suelen estar presentes junto con una desnutrición, pero también aparecer independicntemente como otra manifestación más de un daño de crecimiento y desarrollo fetal. No considerarlas insluidas entre los daños de crecimiento, sobre todo cuando incluso hay un déficit pondo estatural asociado es un error que distorsiona las conclusiones y explica las divergencias en los porcentajes de desnutrición intrauterina señaladas cuando se descartan a los recién nacidos malformados o con disfunciones. Estas desviaciones localjizadas son dependientes del tipo e intensidad dc la noxa, y muy fundamentalmente de la oportunidad en que concurra, pero no de otra diferente a la que ocasiona el daño generalizado. Tanto cste crecimiento integral como el de cada órgano o sistema parece tener un período crítico de mayor sensibilidad, después del cial los daños son esperabies sólo si ellos son de mareada intensidad o de un tipo muy definido de carencia, y está en relación con el período de acelerado crecimiento que en ese momento cada uno ticne. La falta de corrpromiso de los otros, a del crecimiento integral, no puede ser considerado como de diversa patogenia. La mayor frecuencia de malformaciones en $\mathrm{R}$. Nacidos desnutridos demuestra la interrelación de patogenia. Igual conclusión podremos sacar si consideramos que el tipo de malformación y grado de desnutrición fetal que siempre acarrea la rubeola cogestacional es fundamentalmente dependiente del momonto en que se desencadena.

Ha sido suficientemente demostrada la desnutrición de fetos de animales sometidos durante la gravidez a privación de nutrientes. Así por ejemplo, las experiencias de ligadura de vasos principales de una asta uterina en ralas, realizada sólo 7 días antes del parto, pero que abarea $1 / 3$ del período de gravidez. muestra un déficit ponderal y generalizado y de todos los órganos, excepto cl encéfalo, dado que el crecimiento acelerado de este órgano ocurre en estos animales en el período post-natal
(11). Su cumpromiso también cuando se las somete a una privación nutricional durante el períndo de amannantamiento así lo demuestra (12). Avala esta afirmación el compromiso sólo encefálico de los cobayos cuando la noxa interfiere entre los 41 y 45 dias del período de gravidez (13), y de todos los órganos cuando es de mayor duración.

Estas y otras experiencias no muestran analogia con to que acontece en el ser humano, pero sí inducen a buscar la cvidencia demostrativa.

Siendo así de compleja la tarea, y mientras no haya una uniformidad de criterios para abordarla, debcmos contentarnos con la comparación entre poblaciones que dependan de una misma autoridad normativa de evaluación de daños, o que comparen los índices de desviación, o hasta los promedios ponderales, pero entre grupos más homólogos de neonatos; lo que obliga separarlos previamente scgún una misma condición, aplicando cada una o las más importantes de las variables mencionadas. La aplicación de esta metódica bastó para que en un estudio comparativo, rea'izado en Guatemala (14), entre madres con ingestas superiores a 2.200 cal. y otras con inferiores a 1.800 cal. diarias, demostrara diferencias significativas en los pesos promedios de los hijos, agrupados según sexo y madurez de los R. Nacidos, o talla y paridad de las madres. Igualmente en una experiencia de suplementación alimentaria realizada en ese mismo pais (15-16) se concluyó que los hịos de las madres con suplementación de proteínas tenían un peso promedio de nacimiento significativamente superior que un grupo control de igual condición socio-económica y estado nutritivo previo de las madres, si para la comparación se les agrupaba según scxo y paridad. Si además se hubiesen determinado los porcentajas de daños las conclusiones seguramente habrían sido aún más demostrativas.

De igual manera si analizamos el cuadro $N o ̣$ 1, que muestra los hallazgos patológicos o biosociales más comúnmente considerados responsables de un daño nutricional intrauterino del feto, se puede observar que todos cllos excepto los genéticos son derivados de una influencia ambiental negativa, en la que la disminución de mstrientes $y / 0$ oxígeno para el embrión o feto aparce como común denominador. Lal disfunción placentaria no debe ser considerada causal de desnutrición fetal, sino al revés como consecuencia dc un daño del crecimiento y desarrollo del cmbrión, localizado, en el trofoblasto, que acontece con similar patogenia a la del compromiso de cualquier otro órgano. Las experiencias de Winick (17) quc, en base a índices de D. N. A., ha demostráclo una disminución de la celularidad de lás placentas de neonatos dosnutridos, igual que la del resto de los órganos, lo pone aś en evidencia. Un exclusivo mayor com- 
promiso de la placenta acarreará una malformación placentaria responsable de una insuficiencia, que coadyudará o determinará consecuencialmente una desnutrición intrauteriлa; pero la causa primaria será una alteración de crecimiento y desarrollo localizado.

Estas y otras observaciones y experiencias parecen confirmar las conclusiones obtenidas en animales acerca de la real interferencia de las carencias nutricionales de las embarazadas en el determinismo the una desviación del crecimiento y desarrollo fetal. La diferencia sólo está que en ellos por tener períodos de gravidez más breves las noxas interfieren más prolongadamente, los daños se hacen más evidentes y el compromiso es más generalizado. Sólo si acontece durante el período crítico de exclusivo creciniento y desarrollo acelerado de un determinido órgano, el compromiso es sólo localizado; lo que puede ocurrir con mayor probabilidad en el hombre.

\section{U A D R O No 1 \\ HALLAZGOS MAS FRECUENTES EN RELA- CION CON LA DESNUTRICION FETAL INTRAUTERINA}

\section{1.- Hallazgos de orden materno:}

1.1. Talla pequeña.

1.2. Estado nutritivo deficiente.

1.3. Edad avanzada.

1.4. Gran multiparidad o primiparidad.

1.5. Nefropatía gravídica.

1.6. Procesos crónicos hipoxcmiantes.

1.6.1. Asma.

1.6.2. Cardiopatías.

1.6.3. Nefropatías con insuficiencia renal.

1.6.4. Hipertensión o hipotensión.

1.6.5. Anemias crónicas.

1.6.6. Caquexia cancerosa o T.B.C.

1.6.7. Alcoholismo.

1.6.8. Tabaquismo.

1.7. Influencias ambientales.

1.7.1. Bajo nivel socio-económico.

1.7.2. Fatigabilidad física y/o psíquica.

1.7.3. Clima de altura.

1.7.4. Teratógenas. (radiacioncs, fármacos, etc.).

\section{2.- Hallazgos de orden ovular:}

2.1. De la placenta y la envoltura:

2.1.1. Corioamionitis $y / 0$ placentitis.

2.1.2. Infartos, trombosis o hemangiomas.

2.1.3. Fibrinosis difusa.

2.1.4. Placentaciốn gemelar (sindrome pitrabiótico).

2.3.5. Anastomosis arteriovenosas anómalas (gemelos univitclinos).

2.1.6. Placenta extracordial o piatcenta previa.
2.1.7. Deformacisnes de contorno Piacentario.

2.1.8. Desprendimiento prematuro de la platcenta.

2.2. Del cordón:

2.2.1. Inserciones anómalas.

2.2.2. Ausencia de una arteria umbilical.

2.2.3. Circulares apretadas irreductibles.

3. Hallazgos de orden fetal:

3.1. Contaminación ovular por infecciones maternas.

3.1.1. Rubeola cogestional.

3.1.2. Citomegalia cogestional.

3.1.3. Toxoplasnosis cogestional.

3.1.4. Herpes cogestional.

3.2. Factores genéticos constitucionales.

3.3 Aberraciones cromosómicas.

3.4. Malformaciones concomitantes.

En base a estas nucvas experiencias que consideren la interferencia de algunos factores ccológicos condicionantes, a tos ha!lázgos que en seres humanos interrelacionan cansas y efectos de una desnutrición fetal intrauterina, y la analogía con lo que ocurre en los animales sometidos a desnutrición durante su gravidez no puede afirmarse una exclusiva influencia de la desnutrición materna en el estado nutricional de los neonatos, pero sí aseverarse que indudablemente interviene en su determinismo. La demostración ocurrirá cuando se concicrten las voluntades para una acabada investigación, en la que una normalización en tit valoración de los estados de madurez y desviaciones del crecimiento y desarrollo de cada recién nacido se acepte como un requisito previo e includible. Sólo entonces podrán establecerse comparaciones valederas catrc los resultados obtenidos por los diversos investigadores, y medir la real influencia de cada una de las variables condicionantes del curso de un daño nutricional maternofetal.

\section{BiBLIOGRAFía}

1.-Grsenwald, P., Funakan:a, H., Mitand, S., Nishi. mura, $T$. y Takeuchi $S$. lnflucnce of cnviromantial factors on foetal growth in nitn. lancet, 1 : 1026 , 1967.

2.- Simith, C. A. Effect of maternal undermutrition upon the newborn infant in Holland (1944-45) s. Pediat., 30: $229,1947$.

3.- Antonos. A. N. Children born during the siage of Leningrad in 1942. J. Pediat. 30: 250.259, 1947.

4.-Bourquin, A. \& Be'sumem. The preconception dies of women who have had unsuecessful pregnancies. Am, J, Clin. Nutr., 5: 62-69, 1957. 
5.-Thomsou, A. $M$. Diet in pregnancy. Diet in relar tion to the course and outcome of pregnancy. Brit. J. Nutr, 13: 509-515, 1959.

6.- Pathak, $X, L$. Nutritional adaptation to low dictary intakes of calories, proteins, vitamins, and minerals in the tropics. Am. J. Clin. Nutr., 6: 151158,1958 .

7.-McGanify, W. J, O. Cannon, E. B. Bridgforth, $M$. P. Martin, P. M. Densen, J. A. Newbill, S. G. Mc Clean, A, Christie, C, J. Peterson \& W. J. Darby. The Vanderbilt cooperative study of maternal and infant nutrition. Am. J. Obstet. Gynecol., 67: 501527, 1954.

8.- Givovich y Colab. Variación del estado de madurez del neouato Rev. Chil. Ped. Vol. 37, No 7, 1969.

9.--Givovich y colab. Diagnóstico de la desnutrición en el neonato. Rev, Chil. Ped. Vol, 40 No I, 1970.

10.-Rizzardini M. Rev. Ch. Ped. Vol. 44 Nọ 3. $281 \cdot$ 1973.

11.-Wigglesworth, J. S. Experimental growth retardation in the foetal rat. J. Path. Bact., 88: 1, 1964.

12.-Dobbing, J. Vulnerable periods in developing brain. En Applied Neuro-Chemistry. Oxford, Blackwell, 1968 a.
13,-Flexier, L. B. Enzymatic and funtional patterns of the developing mammalian brain. En Waelech. H., dir.: Biochemistry of the Developing Nervous System. New York. Academic Press, 1954.

14.- Evaluación Nutricional de la Población de Centro América y Panamá, Guatemala. Instituto de Nutrición de Centro América y Panamá (INCAP); Oficina de Investigaciones Internacionales de los Jnstitutos Nacionales de Salud (EE. UU.); y Ministerio de Salud Pblica y Asistencia Social, Guatemala, Instituto de Nutrición de Centro América y Panamá, 1969, 136 p. (Publicación INCAP V-25).

15.- tyengar, L. Effect of dietary supplement on birth weight of infunts. En: Firts Așian Congress of Nutrition. Abstracts, Smypasia, Special Reports, Reseurch Communications. Hyderabad, India, January, 28. February 2, 1971. Hyderabad, 20, Kamal Printers 1971. p. 126.

16.- Lechtig, A., J. P. Habicht, E. de León \& G. Guzmán. Influencia de la nutrición materna sobre el crecimiento fetal en poblaciones rurales de Guatemala. II Suplementación alimentaria. Enviado para publicación a Arch. Latinoamer. Nutr.

17.-Winick, $M$. Cellutar growth in human placenta. Pediatric 39: 248, 1969. 